Check for updates

Cite this: Chem. Commun., 2018, 54, 487

Received 16th November 2017 Accepted 9th December 2017

DOI: $10.1039 / c 7 c c 08818 c$

rsc.li/chemcomm

\section{Charge distribution and conformational stability effects of organic structure-directing agents on zeolite synthesis $\dagger$}

\author{
Donghui Jo and Suk Bong Hong (D) *
}

\begin{abstract}
While the use of the so-called organic structure-directing agents (OSDAs) is no stranger to zeolite synthesis at all, the effects of their charge distribution and conformational stability on the phase selectivity of the crystallization remain elusive. To systematically investigate these effects, we have prepared a series of imidazolium and pyrazolium derivatives with the same geometric shape but different positions of nitrogen atoms in the five-membered heterocyclic aromatic ring and used them as OSDAs for the synthesis of zeolites with pure-silica composition. Meaningful differences in the zeolite product were observed.
\end{abstract}

Zeolites and related materials, an extraordinary class of microporous, crystalline solids with regular and well-defined channels and cavities, continue to find new applications in adsorption, separation, and catalysis. ${ }^{1}$ Consequently, there is still need for synthesizing zeolitic materials with novel framework structures. It was in the early 1960s when Barrer first introduced tetramethylammonium into the synthesis mixture of Si-rich zeolite A (framework type LTA). ${ }^{2}$ Since then, the use of alkylamines and alkylammonium ions as OSDAs in zeolite synthesis, both with and without the presence of inorganic species, has enabled the discovery of a great number of unprecedented zeolite structures. ${ }^{3}$ Despite the fact that the geometric correspondence between the organic SDAs and the pore architecture of the final zeolite phase is generally quite loose, on the other hand, it is well established that the framework structure of the zeolite formed can be strongly affected by the shape and size of the OSDA employed. ${ }^{1,3}$ However, there is little known about the effects of their charge distribution and conformational stability on the phase selectivity of the crystallization, ${ }^{4}$ probably due to the lack of OSDA systems suitable for addressing this issue.

Recently, alkylated imidazolium cations, with two nonadjacent nitrogen atoms in the five-membered aromatic ring,

Center for Ordered Nanoporous Materials Synthesis, Division of Environmental Science and Engineering, POSTECH, Pohang 37673, Korea.

E-mail: sbhong@postech.ac.kr

$\dagger$ Electronic supplementary information (ESI) available. See DOI: 10.1039/c7cc08818c have been extensively used as OSDAs in zeolite synthesis and have afforded many materials with novel framework structures or compositions (e.g., zeolites ITW, STW, RTH, etc.). ${ }^{3 d, 5}$ While the pyrazolium derivatives have received little attention as OSDAs, ${ }^{6}$ their aromatic ring with two adjacent nitrogen atoms possesses the same shape, size, and chemical formula as those of the imidazolium ring but a different charge distribution. Therefore, a comparison of the structure-directing abilities of pyrazolium-based cations in zeolite synthesis with those of imidazolium-based cations will allow us to investigate in detail how the charge distribution in OSDAs can influence the phase selectivity. Herein we present the representative products from zeolite syntheses using three pairs of methylated imidazolium and pyrazolium cations as OSDAs to check whether our speculation is valid or not. We have also carried out syntheses using similar imidazolium and pyrazolium derivatives containing an ethyl group and thus some degree of flexibility to show that the conformational stability of OSDAs is another factor affecting the phase selectivity of the crystallization.

Fig. 1 shows a series of alkylated imidazolium and pyrazolium derivatives with the same geometric shape, all of which were synthesized using commercially available compounds (see experimental details in the ESI $\dagger$ ). However, although differences in the position of two nitrogen atoms in their aromatic ring must influence the charge distribution in each pair of OSDAs, identifying differences in their charge distribution is not an experimentally easy task. Thus, we performed density functional theory (DFT) calculations for the structural optimization of each OSDA and then visualized the electrostatic potential (ESP) surface at the B3LYP/6-31+G(d,p) level of theory using the Gaussian 09 program. ${ }^{7}$ The ESP mapping results in Fig. 1 reveal that the blue-colored region near the nitrogen atoms has a higher electrostatic potential energy and thus is more positively charged than the other regions in the molecule, for instance, alkyl groups. More interestingly, there are also differences, both small and large, in the ESP surface (i.e., charge distribution) of these six diazolium pairs, despite the same shape between each pair. 
(a)

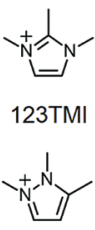

123TMP
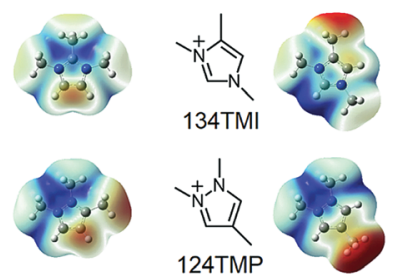

$0.12 \mathrm{Ha}$

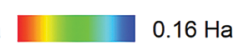

(b)
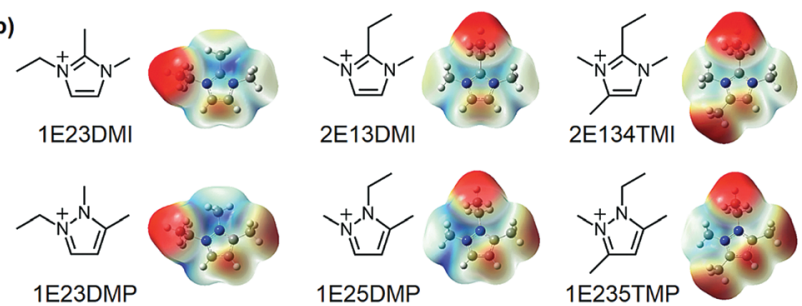

1E25DMP

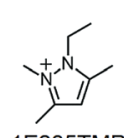

1E235TMP
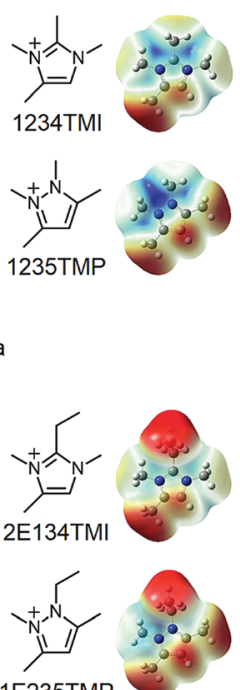

Fig. 1 Imidazolium- and pyrazolium-based OSDAs with (a) only methyl groups and with (b) both methyl and ethyl groups, which have the same geometric shape but different charge distributions, studied in this work. Their electrostatic potentials mapped onto the electron density surface with an isovalue of $4 \times 10^{-4}$, which were calculated at the B3LYP/6-31+G(d,p) level of theory, are also given. Abbreviations: 123TMI, 1,2,3-trimethylimidazolium; 134TMI, 1,3,4-trimethylimidazolium; 1234TMI, 1,2,3,4-tetramethylimidazolium; 123TMP, 1,2,3-trimethylpyrazolium; 124TMP, 1,2,4-trimethylpyrazolium; 1235TMP, 1,2,3,5-tetramethylpyrazolium; 1E23DMI, 1-ethyl2,3-dimethylimidazolium; 2E13DMI, 2-ethyl-1,3-dimethyl-imidazolium; 2E134TMI, 2-ethyl-1,3,4-trimethylimidazolium; 1E23DMP, 1-ethyl-2,3-dimethylpyrazolium; 1E25DMP, 1-ethyl-2,5-dimethylpyrazolium; 1E235TMP, 1-ethyl2,3,5-trimethyl-pyrazolium.

Table 1 lists the products obtained from fluoride-mediated pure-silica zeolite syntheses at $175{ }^{\circ} \mathrm{C}$ and $\mathrm{H}_{2} \mathrm{O} / \mathrm{SiO}_{2}=5.0 \mathrm{using}$ 12 different imidazolium- and pyrazolium-based OSDAs as shown in Fig. 1. The products listed were the only ones obtained in repeated trials, and the pure-silica synthesis composition was selected because the phase selectivity of the crystallization can also be significantly changed by the presence of heteroatoms such as $\mathrm{Al}$ and Ge other than $\mathrm{Si}$ in the synthesis mixture. ${ }^{1,3 c, d}$ ${ }^{1} \mathrm{H}-{ }^{13} \mathrm{C}$ CP MAS NMR spectroscopy evidences that all OSDAs employed here remain intact upon encapsulation within the pores of the zeolite products obtained under the conditions described above (e.g., see Fig. S1, ESI $\dagger$ ). In the case of methylated

Table 1 Representative products from zeolite syntheses using a series of imidazolium and pyrazolium derivatives as OSDAs

\begin{tabular}{llll}
\hline $\mathrm{R}^{a}$ & Product $^{b}$ & $\mathrm{R}$ & Product \\
\hline $123 \mathrm{TMI}$ & ITW & 123TMP & ITW \\
134TMI & ITW & 124TMP & TON + NON + MFI \\
1234 TMI & STW & 1235 TMP & STW \\
1 E23DMI & ITW & 1E23DMP & ITW \\
2 E13DMI & ITW & 1E25DMP & NON \\
2E134TMI & STW & 1E235TMP & HPM-2
\end{tabular}

${ }^{a}$ The composition of the synthesis mixture is $0.50 \mathrm{ROH} \cdot 0.50 \mathrm{HF} \cdot 1.0 \mathrm{SiO}_{2}$. $5.0 \mathrm{H}_{2} \mathrm{O}$, where $\mathrm{R}$ is OSDA, and the abbreviations are the same as those in Fig. 1 . All syntheses were performed under rotation $(60 \mathrm{rpm})$ at $175^{\circ} \mathrm{C}$ for 14 days. ${ }^{b}$ The product appearing first is the major phase. ${ }^{c}$ A layered precursor of zeolite MTF.

diazolium cations, the 123TMI and 123TMP pair always directed the synthesis of zeolite ITW, a two-dimensional (2D) small-pore material. Similarly, both 1234TMI and 1235TMP were found to give the same phase, i.e., STW, a chiral medium-pore zeolite. In contrast, the 134TMI and 124TMP pair shows a notable difference in the phase selectivity of the crystallization: while 134TMI yielded pure ITW, 124TMP gave a mixture mainly made up of TON, a 1D medium-pore material (Fig. S2, ESI $\dagger$ ).

No significant changes in the phase selectivity of the crystallization were observed when zeolite syntheses were carried out at a lower temperature (i.e., $125{ }^{\circ} \mathrm{C}$ ) in the presence of methylated diazolium-based OSDAs (Table S1, ESI $\dagger$ ). As well established, ${ }^{8}$ we also found that an increase in the $\mathrm{H}_{2} \mathrm{O} / \mathrm{SiO}_{2}$ ratio in the synthesis mixture (e.g., from 5.0 to 15.0 ) at $175{ }^{\circ} \mathrm{C}$ could exert a great influence on the phase selectivity (Table S2, ESI $\dagger$ ). While the precise reason that $124 \mathrm{TMP}$ directs the synthesis of impure TON at $175{ }^{\circ} \mathrm{C}$ and $\mathrm{H}_{2} \mathrm{O} / \mathrm{SiO}_{2}=5.0$ is unknown at this time (Table 1), it is worth noting that TON could be synthesized in a pure form using the same OSDA at a higher $\mathrm{H}_{2} \mathrm{O} / \mathrm{SiO}_{2}$ ratio of 15 (Table S2, $\mathrm{ESI} \dagger$ ).

The comparison of the ESP surfaces of 134TMI and 124TMP indicates that the charge distributions of these two cations are significantly different from each other, unlike those of the former two pairs of methylated OSDAs. The region between the two adjacent methyl groups (i.e., blue-colored region) in 124TMP was found to be more positively charged than that near the isolated methyl group (i.e., red-colored region). As shown in Fig. 1a, however, the opposite holds for 134TMI. Given the similarity between 134TMI and 124TMP in many other aspects, including size, shape, total charge, and chemical composition, therefore, the difference in their charge distribution may be responsible for the observed phase selectivity. To our knowledge, this is the first example where the charge distribution effect of OSDAs on their zeolite structure direction is experimentally recognized. Although the precise reason is unclear, it is not very difficult to conjecture that the Coulombic interactions between the OSDA and the negatively charged silicate oligomers during the crystallization process can differ according to the charge distribution in OSDA molecules. This may lead to the formation of different types of organic-inorganic composite structures and thus of different final zeolite structures. Therefore, the use of OSDAs with quite similar molecular shapes but different charge distributions in zeolite synthesis appears to be an alternative route to the search for novel structures.

On the other hand, the ESP surfaces of the three pairs of imidazolium- and pyrazolium-based OSDAs with both methyl and ethyl groups, as shown in Fig. 1b, were found to be not so different from each other. This led us to expect that each pair may produce the same zeolite phase. However, this is only the case for the 1E23DMI and 1E23DMP pair that directs the synthesis of ITW (Fig. S2, ESI $\dagger$ ). As listed in Table 1, 2E13DMI and 2E134TMI gave ITW and STW at $175{ }^{\circ} \mathrm{C}$ and $\mathrm{H}_{2} \mathrm{O} / \mathrm{SiO}_{2}=5.0$, respectively. Also, 1E25DMP and 1E235TMP yielded nonasil (NON) and HPM-2 (i.e., a layered precursor to MTF), ${ }^{5 g}$ respectively (Fig. S2, ESI $\dagger$ ), although we were not able to clearly discriminate between their structure-directing abilities at $125{ }^{\circ} \mathrm{C}$ or $\mathrm{H}_{2} \mathrm{O} / \mathrm{SiO}_{2}=15.0$ (Tables S1 and S2, ESI $\dagger$ ). 
To understand differences in the phase selectivity observed for the latter two pairs of imidazolium and pyrazolium derivatives containing both methyl and ethyl groups, which cannot be explained by differences in the charge distribution, we focused our attention on the effect of a flexible or rotatable bond (i.e., the bond between the aromatic carbon or nitrogen atom and the first carbon atom of the ethyl substituent, denoted as the $\mathrm{C}_{\mathrm{Et}}(3)-\mathrm{E}_{\mathrm{Ar}}(2)$ bond, where $\mathrm{E}$ is $\mathrm{C}$ or $\mathrm{N}$ ) in these OSDAs on the phase selectivity of the crystallization. The relative energy of each OSDA calculated as a function of the $\mathrm{E}_{\mathrm{Ar}}(1)-\mathrm{E}_{\mathrm{Ar}}(2)-\mathrm{C}_{\mathrm{Et}}(3)-\mathrm{C}_{\mathrm{Et}}(4)$ torsion angle at the $\mathrm{B} 3 \mathrm{LYP} / 6-31+\mathrm{G}(\mathrm{d}, \mathrm{p})$ level is shown in Fig. 2a. The flat-shaped conformations with the ethyl group located near the plane of the aromatic ring, whose torsion angles are around $\pm 180^{\circ}$, of $1 \mathrm{E} 23 \mathrm{DMI}$ and 1E23DMP were found to be much more energetically favorable (0.2-4.7 vs. $\left.14.6-19.1 \mathrm{~kJ} \mathrm{~mol}^{-1}\right)$ compared to those of the other two pairs, whereas their flat-shaped conformations with torsion angles around $0^{\circ}$ are more unstable. This can be attributed to the fact that no steric strain by an adjacent substituent is present when the torsion angle of 1E23DMI and 1E23DMP reaches about $\pm 180^{\circ}$. In contrast, 1E25DMP and 1E235TMP showed less preference (19.1 vs. $14.6-15.4 \mathrm{~kJ} \mathrm{~mol}^{-1}$ ) to the flat-shaped conformations at torsion angles around $\pm 180^{\circ}$ than the corresponding imidazolium cations (i.e., 2E13DMI and 2E134TMI) where the two methyl groups are adjacent to the ethyl group attached to the carbon or nitrogen atom of the aromatic ring (Fig. 2a).

It is also remarkable that the shape of the cavities in the four zeolite phases (i.e., ITW, STW, NON, and HPM-2) crystallized using these two pairs of OSDAs, which were found to contain approximately one OSDA molecule per their cavity (Table S3 and Fig. S3, ESI $\dagger$ ), is from flat- to plump-shaped. As a result, the dimension of their height is considerably different from one

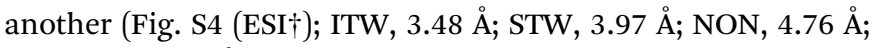
and MTF, $5.46 \AA$ ). Here we compared the cavity dimension of MTF, instead of HPM-2, because, although the structure of the MTF precursor remains unknown, ${ }^{5 g}$ its largest cavity dimension cannot be larger than the interlayer distance in HPM-2. If such is the case, the existence of larger cavities in NON and HPM-2 could then be correlated with the highest energy barrier for the flat-shaped conformations at torsion angles around $\pm 180^{\circ}$ of the OSDAs employed. As shown in Fig. 2a, the flat-shaped conformation of 1E25DMP and 1E235TMP is more unstable than that of 2E13DMI and 2E134TMI, although differences in their energy barrier are 3.8-4.5 $\mathrm{kJ} \mathrm{mol}^{-1}$ (Fig. 2a and Fig. S4, ESI $\dagger)$. Thus, the latter two imidazolium OSDAs may direct the formation of ITW and STW structures consisting of similar flatshaped cavities without much difficulty. This leads us to believe that the structure of the crystallized zeolite product can be influenced by the stability of a particular type of conformation available to the OSDA under zeolite synthesis conditions.

To elucidate the reason behind OSDAs with both methyl and ethyl groups showing differences in the conformational stability, we monitored the $\mathrm{C}_{\mathrm{Et}}(3)-\mathrm{E}_{\mathrm{Ar}}(2)$ bond length in each OSDA as a function of the $\mathrm{E}_{\mathrm{Ar}}(1)-\mathrm{E}_{\mathrm{Ar}}(2)-\mathrm{C}_{\mathrm{Et}}(3)-\mathrm{C}_{\mathrm{Et}}(4)$ torsion angle. As shown in Fig. S5 (ESI $\dagger$ ), the trend of changes in this bond length is essentially the same as that observed for the relative energy of
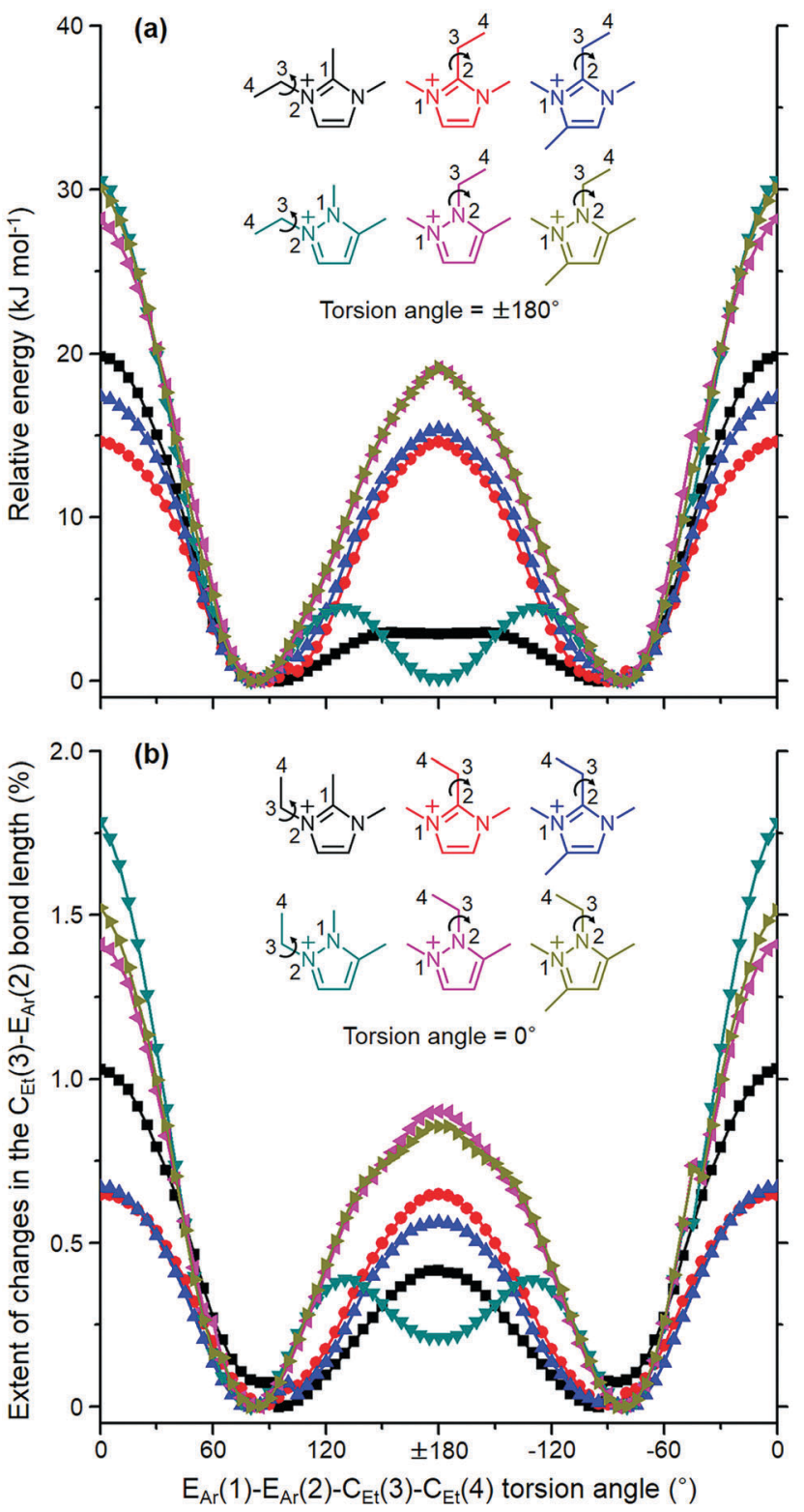

Fig. 2 (a) Relative energies and (b) extents of changes in the $C_{E t}(3)-E_{A r}(2)$ bond length of three pairs of imidazolium and pyrazolium derivatives with both methyl and ethyl groups calculated at the B3LYP/6-31+G(d,p) level of theory as a function of the $E_{A r}(1)-E_{A r}(2)-C_{E t}(3)-C_{E t}(4)$ torsion angle, where $\mathrm{E}$ is $\mathrm{C}$ or $\mathrm{N}$.

each OSDA (Fig. 2a). As expected, in addition, the $\mathrm{C}_{\mathrm{Et}}(3)-\mathrm{C}_{\mathrm{Ar}}(2)$ bonds of 2E13DMI and 2E134TMI are longer than the $\mathrm{C}_{\mathrm{Et}}(3)-\mathrm{N}_{\mathrm{Ar}}(2)$ bonds of the other OSDAs. To directly compare changes in their $\mathrm{C}_{\mathrm{Et}}(3)-\mathrm{E}_{\mathrm{Ar}}(2)$ bond length, we converted them to the unitless measurements that are given with respect to the bond length in the most stable conformation of each of the OSDAs (Fig. 2b). When the torsion angle is ca. $\pm 180^{\circ}$, the extents of changes in the $\mathrm{C}_{\mathrm{Et}}(3)-\mathrm{N}_{\mathrm{Ar}}(2)$ bond length of 1E25DMP and 1E235TMP were calculated to be about $0.9 \%$. However, the $\mathrm{C}_{\mathrm{Et}}(3)-\mathrm{N}_{\mathrm{Ar}}(2)$ bonds in 1E23DMP and 1E23DMI with one adjacent methyl group to their ethyl group are characterized by considerably smaller changes, i.e., $0.2-0.4 \%$. Fig. $2 \mathrm{~b}$ also shows 
that the extent $(0.6 \%)$ of changes in the $\mathrm{C}_{\mathrm{Et}}(3)-\mathrm{C}_{\mathrm{Ar}}(2)$ bond length of 2E13DMI and 2E134TMI is smaller than that $(0.9 \%)$ in the $\mathrm{C}_{\mathrm{Et}}(3)-\mathrm{N}_{\mathrm{Ar}}(2)$ bond length of 1E25DMP and 1E235TMP with the same steric environment near the ethyl group. This indicates that the shorter $\mathrm{C}_{\mathrm{Et}}(3)-\mathrm{N}_{\mathrm{Ar}}(2)$ bond of 1E25DMP and 1E235TMP is more severely elongated to stabilize the flat-shaped conformations, thus reducing their conformational stability more than 2E13DMI and 2E134TMI with a longer $\mathrm{C}_{\mathrm{Et}}(3)-\mathrm{C}_{\mathrm{Ar}}(2)$ bond. Therefore, it is most likely that the conformational stability of these diazolium-based OSDAs can differ according to the type (i.e., $\mathrm{C}$ or $\mathrm{N}$ ) of aromatic ring atom connected to a rotatable ethyl group.

We also compared the relative energies of the theoretical compounds 1-ethyl-2,3,5-trimethylimidazolium (1E235TMI), 4-ethyl-1,3,5-trimethyl-imidazolium (4E135TMI), and 3-ethyl1,2,4-trimethylpyrazolium (3E124TMP) that have the same molecular shape as that of 2E134TMI and 1E235TMP. Here we tentatively defined the torsion angles of the three theoretical OSDAs in a way that their flat-shaped conformations are more stable around $\pm 180^{\circ}$ than around $0^{\circ}$ (Fig. S6, ESI $\dagger$ ). The flat-shaped conformations at torsion angles around $\pm 180^{\circ}$ of 2E134TMI, 4E135TMI, and 3E124TMP containing the $\mathrm{C}_{\mathrm{Et}}(3)-\mathrm{C}_{\mathrm{Ar}}(2)$ bond were found to have lower energy barriers (10.0-15.4 vs. 19.1-20.1 $\mathrm{kJ} \mathrm{mol}^{-1}$ ) than those of 1E235TMI and 1E235TMP with the $\mathrm{C}_{\mathrm{Et}}(3)-\mathrm{N}_{\mathrm{Ar}}(2)$ bond, showing that the energy barriers of OSDAs are again affected by the type (i.e., $\mathrm{C}$ or $\mathrm{N}$ ) of $\mathrm{E}_{\mathrm{Ar}}$ in the $\mathrm{C}_{\mathrm{Et}}(3)-\mathrm{E}_{\mathrm{Ar}}(2)$ bond. It is interesting to note here that their barriers are also relevant to the type of the adjacent (in the direction that the ethyl group is pointing when the torsion angle reaches about $\pm 180^{\circ}$ ) bond in the aromatic ring, i.e., $\mathrm{E}_{\mathrm{Ar}}(2)-\mathrm{E}_{\mathrm{Ar}}(5)$ bond (Fig. S6, ESI $\dagger$ ). As for the $\mathrm{C}_{\mathrm{Et}}(3)-\mathrm{E}_{\mathrm{Ar}}(2)$ bond, 4E135TMI and 3E124TMP with a longer $\mathrm{C}_{\mathrm{Ar}}(2)-\mathrm{C}_{\mathrm{Ar}}(5)$ bond have lower energy barriers (10.0-10.4 vs. $15.4 \mathrm{~kJ} \mathrm{~mol}^{-1}$ ) than 2E134TMI with a shorter $\mathrm{C}_{\mathrm{Ar}}(2)-\mathrm{N}_{\mathrm{Ar}}(5)$ bond.

In summary, a series of imidazolium and pyrazolium derivatives with the same molecular shape but different charge distributions have been used as OSDAs in zeolite synthesis. It is found that differences in the charge distribution of OSDAs as well as in their conformational stability can have a great influence on the phase selectivity of the zeolite crystallization. The overall results of this work may have important implications in better understanding the structure direction effect from OSDAs in zeolite synthesis.

This work was supported by the National Creative Research Initiative Program (2012R1A3A-2048833) through the National Research Foundation of Korea.

\section{Conflicts of interest}

There are no conflicts to declare.

\section{Notes and references}

1 M. A. Camblor and S. B. Hong, in Porous Materials, ed. D. W. Bruce, D. O'Hare and R. I. Walton, Wiley, Chichester, UK, 2011, p. 265.

2 (a) R. M. Barrer and P. J. Denny, J. Chem. Soc., 1961, 971; (b) G. T. Kerr, Inorg. Chem., 1966, 5, 1537.

3 (a) H. Gies and B. Marler, Zeolites, 1992, 12, 42; (b) R. F. Lobo, S. I. Zones and M. E. Davis, J. Inclusion Phenom. Mol. Recognit. Chem., 1995, 21, 47; (c) M. Moliner, F. Rey and A. Corma, Angew. Chem., Int. Ed., 2013, 52, 13880; (d) J. Li, A. Corma and J. Yu, Chem. Soc. Rev., 2015, 44, 7112 .

4 (a) B. M. Lok, T. R. Cannan and C. A. Messina, Zeolites, 1983, 3, 282; (b) Y. Kubota, M. M. Helmkamp, S. I. Zones and M. E. Davis, Microporous Mater., 1996, 6, 213.

5 (a) S. I. Zones, Zeolites, 1989, 9, 458; (b) X. Yang, M. A. Camblor, Y. Lee, H. Liu and D. H. Olson, J. Am. Chem. Soc., 2004, 126, 10403; (c) A. Rojas and M. A. Camblor, Angew. Chem., Int. Ed., 2012, 51, 3854; (d) A. Rojas, E. Martínez-Morales, C. M. Zicovich-Wilson and M. A. Camblor, J. Am. Chem. Soc., 2012, 134, 2255; (e) A. Rojas, M. L. SanRoman, C. M. Zicovich-Wilson and M. A. Camblor, Chem. Mater., 2013, 25, 729; $(f)$ A. Rojas, O. Arteaga, B. Kahr and M. A. Camblor, J. Am. Chem. Soc., 2013, 135, 11975; $(g)$ A. Rojas and M. A. Camblor, Chem. Mater., 2014, 26, 1161; (h) J. E. Schmidt, M. A. Deimund, D. Xie and M. E. Davis, Chem. Mater., 2015, 27, 3756; (i) D. Jo, J. B. Lim, T. Ryu, I.-S. Nam, M. A. Camblor and S. B. Hong, J. Mater. Chem. A, 2015, 3, 19322.

6 P. Wang, Y. Zhao, H. Zhang, T. Yu, Y. Zhang and Y. Tang, RSC Adv., 2017, 7, 23272.

7 (a) P. K. Weiner, R. Langridge, J. M. Blaney, R. Schaefer and P. A. Kollman, Proc. Natl. Acad. Sci. U. S. A., 1982, 79, 3754; (b) A. D. Becke, J. Chem. Phys., 1993, 98, 5648; (c) M. J. Frisch et al., Gaussian 09, revision C.01, Gaussian, Inc., Wallingford, CT, 2010.

8 M. A. Camblor, L. A. Villaescusa and M. J. Díaz-Cabañas, Top. Catal., 1999, 9, 59. 